Revue d'Allemagne et des pays de langue allemande

50-2 | 2018

Humanités environnementales - Quoi de neuf du côté des méthodes?

\title{
Le retour d'expérience (REX) : étude de divers outils techniques dans le cadre de Clim'Ability
}

\section{Alexandre Kudriavtsev}

\section{(2) OpenEdition}

\section{Journals}

Édition électronique

URL : https://journals.openedition.org/allemagne/935

DOI : 10.4000 /allemagne.935

ISSN : 2605-7913

Éditeur

Société d'études allemandes

Édition imprimée

Date de publication : 30 décembre 2018

Pagination : 337-343

ISSN : 0035-0974

\section{Référence électronique}

Alexandre Kudriavtsev, «Le retour d'expérience (REX) : étude de divers outils techniques dans le cadre de Clim'Ability », Revue d'Allemagne et des pays de langue allemande [En ligne], 50-2 | 2018, mis en ligne le 30 décembre 2019, consulté le 18 mai 2021. URL : http://journals.openedition.org/allemagne/935 ; DOI : https://doi.org/10.4000/allemagne.935 


\section{Le retour d'expérience (REX): étude de divers outils techniques dans le cadre de Clim'Ability}

\section{- Alexandre Kudriavtsev*}

L'évaluation des risques et des vulnérabilités d'une entreprise (organisation) au changement climatique constitue une étape centrale dans un processus d'adaptation au changement climatique. Il en est ainsi car il s'agit d'un moment fondateur de l'accession à de multiples informations et à la structuration de différents types de connaissance. Nous revenons dans cet article sur les enjeux de la conception d'outils de diagnostic et de leur passation.

Cet article vise à comprendre en quoi le changement de la configuration du réseau rassemblant des humains (usagers) et des non-humains (objets techniques) par l'adjonction d'un nouvel intermédiaire humain modifiera les résultats de la boîte à outils Clim'Ability?

Actuellement, les outils d'aide à la décision qui visent à sensibiliser les entreprises aux effets du changement climatique et à stimuler leur comportement d'adaptation ont le vent en poupe. Ils font l'objet d'un fort investissement de recherche \& développement. Peut-on pour autant les considérer comme des «objets-frontières»? Pour ce faire, il faut qu'ils contribuent a minima au déplacement des points de vue des acteurs ${ }^{(1)}$. On cherche à savoir si ce déplacement se fera plus facilement quand le réseau d'acteurs "humains-outils" se transforme grâce à la présence d'un intermédiaire humain. Pour comprendre cela nous avons tout d'abord étudié les enjeux du diagnostic pour une entreprise. Par la suite, nous nous sommes appuyés sur la sociologie de la traduction afin d'identifier et d'analyser les forces actantes dans le réseau "humains-outils». Enfin, nous présentons quelques expériences d'application des outils construits dans le cadre du projet Clim'Ability.

* Chercheur dans le projet Clim'Ability Interreg V, INSA de Strasbourg.

1 Geoffrey C. Bowker, Susan Leigh Star, Sorting Things Out: Classification and Its Consequences, Cambridge (Mass.)/London, the MIT Press, 1999. 


\section{Outils de diagnostic. Quelle utilité pour une entreprise?}

La problématique de la conception et de l'utilisation d'outils de diagnostic comme des objets techniques n'est pas récente. Elle est en revanche davantage discutée depuis quelques années avec la multiplication d'événements extrêmes provoqués par le changement climatique. Ceux-ci ont entraîné de lourdes pertes, non seulement économiques mais également humaines.

La sensibilisation d'une entreprise (organisation) à travers l'outil de diagnostic permet une meilleure connaissance du contexte local, la prise en charge la plus juste des risques climatiques et la construction de la résilience cognitive des décideurs. Pour cette raison, les mesures d'adaptation aux changements climatiques $(\mathrm{CC})^{(2)}$ nécessitent une approche contextuelle de terrain pour laquelle les entreprises disposent de diverses stratégies.

Afin de mieux comprendre les causes et effets des CC, il convient d'identifier les solutions circonstanciées. Ainsi l'analyse des risques et des vulnérabilités fournit des informations qui alimentent la prise de décision qui, si la bonne volonté est là, se transforme en action efficace et efficiente.

L'analyse des risques actuels et passés constitue donc un premier pas dans l'identification des risques futurs. Les mesures qui réduisent les vulnérabilités ne sont pas toutes nécessairement adaptées aux évolutions du climat. Il existe une grande palette d'outils d'aide à la décision, endogénéisant tant les aspects économiques, qu'environnementaux du CC.

Les outils d'aide à la décision dédiés à l'analyse des risques climatiques peuvent aider à

- sensibiliser les utilisateurs et affiner leur connaissance des aléas, des risques, des vulnérabilités et des capacités actuelles d'adaptation;

- identifier des vulnérabilités actuelles et futures;

- traiter de façon systématique les aléas et risques climatiques;

- évaluer les risques et développer des stratégies d'adaptation aux CC;

- promouvoir les liens entre scientifiques, décideurs et autorités et collectivités locales.

\section{Réseau d'acteurs «humains-outils»}

Les fondateurs de la théorie de la traduction décrivent la traduction des objets techniques comme une interaction entre le réseau d'acteurs "humains-outils» dans lequel se produisent des transformations à la suite de différentes épreuves ${ }^{(3)}$. Ces transformations sont analysées en tant qu' «inscription» pour un créateur et "description » pour un usager ${ }^{(4)}$. Les actions, comme le test technique d'outil, l'association d'autres acteurs, le test auprès des usagers, peuvent être interprétées comme «une confrontation entre

2 CC: Changements qui sont attribués directement ou indirectement à une activité humaine altérant la composition de l'atmosphère mondiale et qui viennent s'ajouter à la variabilité naturelle du climat observée au cours de périodes comparables. Convention-cadre des Nations unies sur les changements climatiques (CCNUCC), 1992.

3 Michel Callon, "Éléments pour une sociologie de la traduction. La domestication des coquilles Saint-Jacques et des marins dans la baie de Saint-Brieuc», L’année sociologique, $\mathrm{n}^{\circ} 36$ (1986), p. 170208; Madeleine Aкrich, «Les formes de la médiation technique», Réseaux, nº 60 (1993), p. 87-98.

4 M. Akrich, ibid. 
l'environnement inscrit dans le dispositif et l'environnement décrit par son déplacement du dispositif ${ }^{(5)}$. Suite aux confrontations, les dispositifs techniques, les acteurs et les autres éléments se trouvent transformés, renégociés et de nouvelles formes d'organisations et de savoirs sont produites.

Dans le processus cyclique de "boucles itératives» (modèle tourbillonnaire de l'innovation), les objets intermédiaires jouent le rôle de catalyseur pour l'appropriation. Mais ce modèle nécessite compromis et capacité d'adaptation et par conséquent peut conduire le dispositif technique à redéfinir ses propriétés et ses usagers.

En même temps, l'appropriation est fortement influencée par la négociation et la médiation autour des objets-frontières qui participent directement au processus d'intéressement des acteurs. L'appropriation est un processus itératif dans lequel les propriétés et les actions récurrentes peuvent être considérablement modifiées à la suite de compromis et de négociations. Grâce à son aspect réflexif et évolutif tant les acteurs que les dispositifs ou encore les organisations sont régulièrement amenés à en redéfinir les modalités.

Selon Vinck ${ }^{(6)}$, les notions «d'objet-frontière» et «d'objet intermédiaire» sont à dissocier car elles proviennent de recherches différentes. L'objet-frontière est apparu avec l'analyse des intersections entre différents mondes sociaux autour d'un lieu spécifique, un musée d'histoire naturelle. Les «objets intermédiaires», quant à eux, sont qualifiés comme "des entités physiques qui relient les humains entre eux» ${ }^{(7)}$. L’enquête portait sur l'étude de 120 réseaux de coopération scientifique ${ }^{(8)}$ dans le domaine de la santé.

La définition en est bien différente puisqu'elle confère aux objets intermédiaires un rôle de marqueur temporel et d'opérateur de changement. Divers travaux de recherche en sociologie des sciences et des techniques ${ }^{(9)}$ en reprennent le vocable. Des objets intermédiaires peuvent aussi devenir des objets-frontières ${ }^{(10)}$ notamment lorsqu'ils sont équipés, par exemple, de métadonnées, qui rendent possible la constitution d'une équivalence entre des mondes hétérogènes ${ }^{(11)}$. Quant à la différence mais aussi aux glissements possibles entre les objets-frontières et les objets intermédiaires, «[I]ls [ici des objets intermédiaires] sont des objets-frontières lorsque des éléments structurels

5 Madeleine Aкrich, Les formes de la médiation technique. Réseaux, Paris, La Découverte, 1993, p. 87-98.

6 Dominique VINCK, «De l'objet intermédiaire à l'objet-frontière», Revue d'Anthropologie de connaissances, 3/1 (2009), p. 210.

7 Dominique VINCK, «Les objets intermédiaires dans les réseaux de coopération scientifique. Contribution à la prise en compte des objets dans les dynamiques sociales », Revue Française de Sociologie, XI (1999), p. 385-414, ici p. 392.

8 Dominique Vinck, Du laboratoire aux réseaux. Le travail scientifique en mutation, Luxembourg, Office des Publications officielles des Communautés européennes, 1992, 510 p.; D. VINCK, «Les objets intermédiaires dans les réseaux de coopération scientifique» (note 7).

9 Bruno Latour, Aramis ou l'amour des techniques, Paris, La Découverte, 1992, 248 p.; Madeleine Akrich, Michel Callon, Bruno Latour, Sociologie de la traduction. Textes fondateurs, Paris, Presses Mines (Sciences sociales), 2006, $401 \mathrm{p}$.

10 Leigh STAR, Boundary objects and beyond: Working with Leigh Star, Cambridge (Mass.), The MIT Press, 2015, $559 \mathrm{p}$.

11 Bowker/Star, Sorting Things Out: Classification and Its Consequences (note 1). 
(dans ou associés à l'objet ou à un ensemble d'objets) sont partiellement communs à plusieurs mondes sociaux» ${ }^{(12)}$.

Tous les supports que nous utilisons dans Clim'Ability sont susceptibles d'être analysés sous l'angle «d'objet-frontière», comme «Clim'Ability Check» ou «Clim'Ability Diag», outils d'analyse des risques climatiques, la cartographie de type SIG, la représentation multidimensionnelle des risques et la dissémination des résultats des recherches scientifiques.

Tout ceci nous permet de nous demander dans quelle mesure ces objets techniques, qui sont aussi des objets-frontières, participent à la construction de compromis, de savoirs partagés entre les acteurs et visent à faciliter les échanges entre les scientifiques et le monde de l'entreprise, en favorisant un passage à l'action.

\section{Retour d'expérience: mini-questionnaire Clim'Ability}

Afin de pouvoir rapidement tester le positionnement d'une entreprise par rapport aux risques climatiques, un mini-questionnaire de sept questions a été conçu. Nous avons testé celui-ci durant plusieurs salons professionnels, en France et en Allemagne, consacrés à diverses thématiques, comme la logistique (SITL), la santé au travail, la sécurité des entreprises (Preventica) ou encore les technologies embarquées et l'informatique (Embedded World).

Le choix des salons comme terrain d'étude répond à deux hypothèses:

1) L'accès aux entreprises est direct, sans obstacles habituels (prise des rendez-vous, relances, échanges des mails, etc.) et doit faciliter la communication et la collecte ultérieure des informations.

2) Souvent les exposants s'ennuient et sont prêts à avoir des échanges, ce qui permet de libérer la parole informelle et de récolter de précieuses informations.

Dans un premier temps, nous approchions les exposants avec une brève introduction de Clim'Ability pour ensuite leur proposer de remplir un court questionnaire. Très vite nous avons dû changer notre approche car majoritairement les exposants n'étaient pas prêts à remplir le questionnaire, pour des raisons peut-être éthiques ou par absence d'envie. Nous avons donc décidé de remplir le questionnaire nous-même à partir d'échanges directs avec les exposants. Cela a permis de régler le problème en faisant augmenter le taux de répondants, non sans quelques répercussions imprévues.

En effet, quand un chercheur se saisissait du questionnaire et commençait à poser des questions en prenant des notes, le désintéressement de l'exposant était presque instantané. Comme si cette feuille qu'un chercheur tenait devant lui créait une rupture relationnelle. Visiblement, ce passage obligatoire opérait une transition du mode de dialogue informel, caractérisé par l'échange réciproque (same eye level), au mode d'entretien formel suivi par le transfert des rôles et des pouvoirs. Cette nouvelle configuration s'est donc souvent caractérisée par l'échange unilatéral du type «solliciteurdonneur » conduisant la relation à une fin anticipée et à peu de libération de la parole.

Il est probable que, pour une part, les réponses évasives et la piètre qualité de la relation en mode d'entretien étaient dues au fait que les bénéfices de ce «don», en 
temps et partage de connaissances (éventuellement considérées par l'exposant comme confidentielles), n'étaient pas explicites pour l'entreprise ou «l'exposant rationnel».

Cette seconde tentative plus ou moins réussie fut suivie par une nouvelle procédure: nous avons délibérément décidé de dissimuler le questionnaire et de poser les questions sous forme d'échange informel. À la fin de l'échange, le chercheur finissait par expliquer les objectifs de la démarche en faisant apparaître le questionnaire. Ensuite, celui-ci était chargé de le compléter en présence de l'exposant, qui devait valider la fidélité des réponses retranscrites.

Cette approche déguisée, mais nécessaire, nous a permis d'augmenter encore le taux de réponse, tout en augmentant leur qualité et leur exhaustivité; chose impossible dans la première situation d'entretien.

\section{Retour d'expérience: Clim'Ability Check}

Clim'Ability Check est un outil d'autodiagnostic et de sensibilisation des entreprises aux risques provoqués par le changement climatique. L'outil se présente sous forme d'un questionnaire en ligne comprenant une quarantaine de questions qui couvrent diverses thématiques comme le contexte géographique, les activités principales d'une entreprise (la logistique, la production, l'approvisionnement, etc.), les risques climatiques, l'évolution future de la réglementation et ses multiples impacts positifs et négatifs.

Un facteur multiplicatif est ensuite appliqué à la sensibilité climatique auto-évaluée par l'utilisateur, selon des paramètres liés à la température et aux précipitations (16 paramètres climatiques en tout, incluant les précipitations saisonnières, la moyenne annuelle de température, les vagues de chaleur, les périodes de sécheresse, etc.).

Ce facteur multiplicatif est calculé sur de petits territoires homogènes au niveau des écosystèmes naturels, appelés «Naturräume». Les Naturräume sont répartis en zones sur toute l'Allemagne et constituent une référence géographique pour «KlimaFolgenCheck». Lorsque l'utilisateur indique son code postal, l'outil (KlimaFolgenCheck) peut positionner son entreprise dans un «Naturraum» et définir, à partir d'un tableau enregistrant les paramètres climatiques, le coefficient multiplicateur d'évolution du climat futur:

- 1 si pas d'évolution dans les projections à l'horizon 2050 pour les scénarios RCP4.5 et 8.5 ,

- 1,25 si un seul des scénarios montre une évolution positive,

- 1,5 si les deux scénarios montrent une évolution positive.

À l'issue de l'évaluation, l'entreprise reçoit un bilan qui récapitule ses vulnérabilités et risques et propose des recommandations générales.

Clim'Ability Check résulte d'un travail à partir de l'outil allemand KlimaFolgenCheck conçu par l'Institut für Technologie und Arbeit (Kaiserslautern) et Rheinland-Pfalz Kompetenzzentrum für Klimawandelfolgen, et en partenariat avec ses concepteurs. Plus de 30 utilisateurs ont complété ce questionnaire en ligne. Pourtant le taux de non-réponses y est élevé: 25-35\% des questions sont restées sans réponse.

En France, l'outil a été testé auprès de 6 entreprises du Port autonome de Strasbourg lors d'entretiens semi-directifs. Certes, six entretiens ne peuvent être considérés comme un échantillon suffisant pour l'analyse quantitative profonde, mais fournissent cependant quelques idées intéressantes. 
Parmi les premières choses que nous avons remarquées se trouve l'évolution du taux de réponse et la diminution des questions sans réponse. Bien sûr, quel que soit le mode de gestion d'un questionnaire (en ligne ou face à face), il existe de nombreux impacts susceptibles de biaiser des réponses. Nous avons donc gardé en tête les quatre exigences scientifiques nécessaires inhérentes au questionnaire ${ }^{(13)}$ :

- compréhension de la question

- rappel des informations demandées (mémoire)

- évaluation du lien entre l’information récupérée et la question

- réponse.

Pourtant le constat fut clair: la manière de présenter un questionnaire (par exemple mode auditif, oral, visuel) affectait des facteurs comme le taux de réponse aux questions, le décrochage précoce, l'incompréhension des questions considérées trop complexes, vagues ou personnelles. Chaque mode proposant différents degrés d'accompagnement change complètement la manière de collecter les informations et par conséquent impacte la qualité des données.

\section{Conclusion}

Lors de tous les entretiens nous avons ainsi observé que la présence d'un intermédiaire humain aidait à faire émerger des narrations, des petits récits, la mobilisation de la mémoire des événements marquants. Cela nous a donc permis d'avoir un panorama plus substantiel de l'impact positif de la situation d'entretien sur la qualité des données recueillies.

En général, lors de la conception d'un objet technique, les usagers ont peu de pouvoir et le mode d'interaction est déterminé par les créateurs. Nous caractérisons notre approche comme top-down quand l'objet technique va configurer son usager. Selon Patrice Flichy, «la conception et la production d'un nouvel objet nécessite de définir l'identité des usagers potentiels, et d'établir les contraintes de leurs futures actions » ${ }^{(14)}$. Dans cette logique, l'objet technique va programmer l'usager et inciter certains modes d'utilisation plus que d'autres. En même temps, Stuart Hall ${ }^{(15)}$ montre que l'usager d'un objet technique «peut soit ne suivre qu'une partie des inscriptions du message, soit rejeter en bloc le message». Cet aspect de rejet est un révélateur de rééquilibrage de pouvoir qui n'apparaît qu'au moment du travail sur le terrain et capable de perturber des objectifs de sensibilisation.

Construisant nos outils, nous visons à sensibiliser les entreprises sur le changement climatique et à les inciter à passer à l'action. Cependant, nous avons pu observer que dans beaucoup de cas l'usager d'un outil de diagnostic peut «se rebeller» contre certains blocs d'analyse en altérant les résultats voulus de l'interaction. Il apparaît que cet effet négatif pourrait être atténué par l'association des usagers au stade de la conception, dès les premières étapes avec l'établissement du cahier des charges. Les approches du type bottom-up devront être explorées d'avantage pour exploiter cette piste.

13 Roger Tourangeau, "Cognitive Sciences and Survey Methods», Cognitive Aspects of Survey Methodology: Building a Bridge Between Disciplines, Washington (DC), National Academy Press, 1984.

14 Patrice Flichy, «Technique, usage et représentations», Réseaux, n 148-149 (2008), p. 147-174, ici p. 148.

15

Stuart Hall, «Codage, décodage», Réseaux, n 68 (1994), p. 27-39, ici p. 30. 


\title{
Résumé
}

L'évaluation des risques et des vulnérabilités d'une entreprise (organisation) au changement climatique est une étape centrale dans un processus d'adaptation au changement climatique. À travers cette première étape, l'adaptation au changement climatique permet à une entreprise de réduire les impacts négatifs et les coûts associés au changement climatique, tout en tirant parti des nouvelles opportunités découlant de ces changements. Cette première étape fournit un cadre pour aider une entreprise à concevoir sa propre stratégie d'adaptation basée sur les connaissances générées, rassemblées et synthétisées provenant de diverses sources d'expertise interne, et aussi externe. Nous étudions ici les problèmes de la conception d'outils d'auto-évaluation et de l'expérience utilisateur finale. Le réseau d'acteurs "humains-objets techniques" peut être transformé par la présence d'un intermédiaire humain. Cet article a pour objectif d'explorer comment les résultats de la boîte à outils Clim'Ability seront modifiés par l'ajout d'un intermédiaire humain à la configuration du réseau «humain-objet technique».

\begin{abstract}
Assessing the risks and vulnerabilities of a company (organization) to climate change is a central step in a process of adaptation to climate change. Through this first step, climate change adaptation enables a company to reduce the negative impacts and costs triggered by climate change, while taking advantage of new opportunities arising from these changes. This first step provides a framework to help a company design its own knowledge-based adaptation strategy that is generated, gathered and synthesized from various sources of internal as well as external expertise. Here we study the design issues of self-assessment tools and related end-user experience. The network of actors "human-technical object» can be transformed by the presence of a human intermediary. This article aims to explore how the results of the Clim'Ability toolbox will be modified by adding a human intermediary to the configuration of the "human-technical object» network.
\end{abstract}

